refinement by providing sample and hold facilities. By this means the angle meter may be watched for coincidence with the trial value and, when steady, the $V_{\mathrm{a}}$ and $V_{s}$ values may be held for say $20 \mathrm{sec}$. while the readings are noted.

Mention was made earlier of the proximity of the $V_{\mathrm{a}}$ and $V_{\mathrm{mg}}$ peaks and it follows from the geometry that the higher the wind velocity, the closer the peaks become. At what point this occurs in the wind scale depends upon the shape of the polar curve and the size of the yacht. It has been observed that up to the point where the maximum velocity for the yacht has been realized the $V_{\mathrm{mg}} / V_{\mathrm{t}}$ curve is linear and that beyond this point performance apparently tends to fall off. The point where the $V_{\mathrm{a}}$ meter alone may be used to indicate the best course will have to be a matter of individual subjective determination. That the effect does occur can be seen from a manipulation of the $V_{\mathrm{mg}} / V_{\mathrm{t}}$ equation, which may be re-written :

$$
\left(2 V_{\mathrm{mg}} / V_{\mathrm{t}}\right)+\mathrm{I}=\left(V_{\mathrm{a}}^{2}-V_{\mathrm{s}}^{2}\right) / V_{\mathrm{t}}^{2}
$$

from which it will be seen that, as $V_{\mathrm{a}}$ becomes larger and $V_{\mathrm{s}}$ attains its maximum value, $V_{s}$ cannot substantially alter the angle $\alpha$ at which $V_{\mathrm{mg}}$ becomes a maximum, i.e. when $V_{\mathrm{a}}$ also becomes a maximum. In the downwind case, where the polar curve may have an inflexion as shown in Fig. 1 , the maximum value of $V_{\mathrm{mg}}$ occurs when $V_{\mathrm{s}} / V_{\mathrm{a}}$ is at a maximum. How this happens will be readily seen from Fig. 6 which is an enlarged portion of the downwind area.

In conclusion it must be said that the $\beta$ meter should have a scale capable of indicating $\mathrm{I}^{\circ}$ increments. The other two velocity instruments could profitably be repeating instruments with sample and hold facilities. The $V_{\mathrm{a}}$ repeater would also benefit the user if an expansion (i.e. non-linear) amplifier, with offset and a qualitative scale, could be brought into commission once the sailing conditions warrant its use as a $V_{\mathrm{mg}}$ meter.

The author is grateful to the Board of EMI Marine for permission to publish this work.

\title{
The Use of ABC Tables in Preparing for Star Observations
}

\author{
Captain P. A. Thompson
}

As part of their navigational outfit many of the ships in service today are equipped with Sight Reduction Tables (A.P. $3270=$ H.O. 249), and many navigating officers include amongst their personal kit a Rude Star Identifier (H.O. 2102), or some similar device according to individual preference, for the purpose of obtaining the approximate altitude and azimuth of a selected body as a preliminary to making observations, particularly for star sights. However, for the benefit of those without such means at their disposal and as a matter of interest for others, the use of $A B C$ tables for this purpose is suggested. The origin and author of this method are not known and it does not appear to be included in any of the navigational manuals which have been in circulation in recent years, but there 
was reference to it by Capt. S. T. Lecky in his General Utility Tables first published in 1897 , a reference for which I am indebted to Capt. C. H. Cotter.

This provides a quick and simple way of obtaining the azimuth and altitude of a body within sufficiently close limits for pre-setting the sextant, a feature of especial value when taking star sights during periods of cloudy weather when being ready can make the difference between getting observations or remaining dependent on a D.R. position. For those navigating small craft where stowage space is at a premium, both the preparatory and final working can be done using one set of tables.

The details following are for the use of the $A B C$ tables in both Norie's and Burton's Nautical Tables, those most commonly used in merchant ships, and the equivalent tables in Myerscough and Hamilton's Rapid Navigation Tables.

Norie's $A B C$ Tables: Calculate the azimuth for the selected star in the usual manner.

For altitude: Enter Table A at top or bottom of page using azimuth instead of hour-angle, and with latitude at side, take out quantity $A$.

Enter Table $\mathrm{C}$ with latitude in side column and moving laterally to find hour angle, take $\mathrm{C}$ from head of this column.

Combine $\Lambda$ and $C$. Take the sum if Lat. and Decl. are the same name, unless the Decl. is less than the Lat.; take the difference in all other cases.

Enter Table B with azimuth at head or foot of column, follow down column to find $\mathrm{AC}$; the altitude will be found abreast of this quantity in Decl. column.

Burton's ABC Tables: Calculate azimuth of star in the usual manner.

For altitude: Enter Table $A$ with azimuth at head of page and latitude at side, take out quantity $A$.

Enter Table $\mathrm{C}$ with hour-angle at head of page and latitude at side, take out quantity $\mathrm{C}$.

Combine $\mathrm{A}$ and $\mathrm{C}$ following rules as above for Norie's Tables.

Enter Table B with azimuth at head of page, follow down column to find quantity $\mathrm{AC}$; the altitude will be found abreast of this quantity in column headed Decl.

Rapid Navigation Tables (Myerscough and Hamilton): Calculate azimuth in the usual manner.

For altitude: With azimuth at head of page and latitude in side column, take out quantity $\mathrm{X}$.

With latitude at head of page, find hour-angle in azimuth column and extract quantity $\mathrm{Z}$.

Combine $\mathrm{X}$ and $\mathrm{Z}$ according to rules for $\mathrm{A}$ and $\mathrm{C}$.

With azimuth at head of page, proceed down column $Y$ to find quantity $\mathrm{XZ}$; the altitude will be found abreast of this figure in column headed Lat/ Alt.

\section{Examples}

Posn. $20^{\circ} 00^{\prime}$ N., $65^{\circ} 00^{\prime}$ E. 1800 hrs. Local Time (1 300 hrs. G.M.T.)

Altitude required to observe Vega, Antares, and Regulus.

Calculated azimuth of each body:

Vega $054^{\circ}$; Antares $131^{\circ}$; Regulus $266^{\circ}$; 
Using Norie's $A B C$ Tables:

$\begin{array}{cllll} & & \text { Vega } & \text { Antares } & \text { Regulus } \\ \text { Table A } & 0.26 & 0.32 & 0.03 \\ \text { " C } & 0.11 & 0.83 & 1.12 \\ \text { Ät. B } & 0.37 \text { (Sum) } & 0.51 \text { (Diff.) } & 1.09 \text { (Diff.) } \\ \text { Alt. } & & 163^{\circ} & 21^{\circ} & 47 \frac{1}{2}^{\circ}\end{array}$

Using Rapid Navigation Tables:

\begin{tabular}{|c|c|c|c|}
\hline Col. X & $2 \cdot 64$ & $3 \cdot 16$ & 0.25 \\
\hline,$\quad \mathbf{Z}$ & $I \cdot I 2$ & $8 \cdot 3 \mathrm{I}$ & I I $\cdot 20$ \\
\hline Ali" & $\begin{array}{l}3 \cdot 76 \text { (Sum) } \\
17^{\circ}\end{array}$ & $\begin{array}{l}5.15 \text { (Diff.) } \\
21^{\circ}\end{array}$ & $\begin{array}{l}10.95 \text { (Diff.) } \\
47 \frac{1}{2}\end{array}$ \\
\hline
\end{tabular}

Using Sight Reduction Tables (AP 3270 ):

Vega: Az. $054^{\circ}$, altitude $16^{\circ} 55^{\circ}$

Antares: Az. $131^{\circ}$, altitude $21^{\circ} 34^{\prime}$

Regulus: Az. $266^{\circ}$, altitude $47^{\circ} 46^{\prime}$

\section{Irradiation: A Note}

\section{Charles H. Cotter}

IT is some considerable time since the questions associated with irradiation were considered in the pages of the Journal. Indeed the interesting and practical paper 1 by Captain Brett Hilder is the latest considerable contribution to the Journal and this appeared no less than twenty years ago. It is only in comparatively recent times that nautical astronomers have been provided with an authoritative irradiation correction of any sort. This, which applies only to observations of the Sun's upper limb, was incorporated in the altitude correction table for the Sun and appeared annually in the Nautical Almanac after 1953, the year of Hilder's paper, but was discontinued after 1969 .

The introduction of the irradiation correction stemmed from the work of Clemence on atmospheric refraction, some of the results of which were published in 1951.2 It was Clemence's paper which appears to have prompted Hilder to record his ideas on the subject. Haines and Allen in their paper ${ }^{3}$ of 1968 state that most of the experimental evidence to date suggests that the cause of irradiation is 'the scattering of light within the observer's eye'. This it seems produces an increased minimal angle of resolution (MAR), the determination of which Haines and Allen used to quantify the irradiation phenomenon. The implication in their statement is that the cause, or causes, of irradiation are not completely understood.

The earliest reference to irradiation that $I$ have found in a navigational manual is in the sixth edition (I have checked that it is not in the first) of Raper's Practice. ${ }^{4} \mathrm{He}$ makes a very brief mention:

'The effect of irradiation, or the increase of the Sun's apparent diameter, caused by the extreme brightness, and which may amount to $5^{\prime \prime}$ or $6^{\prime \prime}$ (Dr. 